\title{
El trabajo como fuente de resultados. Hacia una conceptualización más integrada del diagnóstico del enriquecimiento del trabajo en América latina*
}

\author{
Work as a Source of Results. Towards a More \\ Integrated Conceptualization of the Diagnosis of \\ Work Enrichment in Latin America
}

RECIBIDO: 14 DE FEBRERO DE 2020/ACEPTADO 24 DE JUNIO 2020

\section{MARÍA ANDREA GENOUD \\ Eseade (Escuela superior de Economía y Administra- ción de Empresas) \\ info@cofundal.com}

Resumen: El trabajo describe el funcionamiento de una Herramienta de Diagnóstico que busca Enriquecer las Funciones Laborales (HDEFL) en América Latina, adaptada del Modelo de las características del puesto (MCP) de Hackman y Oldham (1974 y sus diversas revisiones) y con base en la investigación sobre estructuras laborales realizada durante 10 años en Argentina por Genoud, Broveglio y Picasso (2014, 2018). El enfoque integrador interdisciplinario de diseño del trabajo permite "desmenuzar" qué características del diseño del trabajo (CDT, variables motivacionales, sociales y contextuales), activan tres estados psicológicos críticos (EPC), condicionando los resultados del trabajo (RT) en los trabajadores, en términos de comportamiento, actitudes y bienestar.

La tipología del estudio es exploratoria, descriptiva y explicativa; se busca alentar cortes interpretativos transdisciplinarios anclados en la Sociología del Trabajo, las ciencias de la Administración y la Psicología Organizacional.

Palabras clave: Enriquecimiento del trabajo, trabajo significativo, comportamiento humano, trabajo en equipo, satisfacción laboral.

\section{GERARDO DARÍO BROVEGLIO \\ Uade (Universidad Argentina de la empresa) gbroveglio@uade.edu.ar}

Abstract: This work describes how the Diagnostic Tool that seeks to Enrich Labor Functions (HDEFL), works in Latin America, adapted from the Model of the Job Characteristics Theory (MCP) of Hackman and Oldham (1974 and its various reviews) and based on research on labor structures carried out during 10 years in Argentina by Genoud, Broveglio and Picasso (2014, 2018). The interdisciplinary integrative approach to work design, allows to identify what characteristics of work design (CDT, motivational, social and contextual variables), activate three critical psychological states (EPC), conditioning the results of work (RT) in workers, in terms of behavior, attitudes and well-being. The typology of the study is exploratory, descriptive and explanatory; it seeks to encourage transdisciplinary interpretative cuts in the organizational studies anchored in the Sociology of Work, Administration sciences and Organizational Psychology.

Key words: Work Enrichment; Meaningful Work; Human Behavior; Team Work; Work Satisfaction 
Las personas construyen un conjunto de creencias, valores y actitudes hacia el trabajo a lo largo de sus vidas; en este sentido el significado del mismo va mutando a través de las experiencias subjetivas de socialización y a partir de las situaciones contextuales que se producen, alternativamente, en la organización laboral y en la coyuntura global en que se lo realiza ${ }^{1}$. Numerosos autores han abordado el análisis del significado del trabajo asumiendo que es un constructo multidimensional complejo ${ }^{2}$, cuya configuración supone estudiar aspectos diversos como su centralidad (absoluta y relativa), las normas éticas personales y sociales que lo rigen, los valores que las personas esperan capitalizar en el trabajo y su congruencia con los que promueven las organizaciones, los aspectos descriptivos del trabajo, su vinculación con los resultados de la función laboral's.

El enfoque integrador interdisciplinario del diseño del trabajo permite "desmenuzar" qué características motivacionales, sociales y contextuales de cada trabajo son centrales en el diseño del trabajo a realizar (CDT), para luego relacionar a través de una herramienta de diagnóstico, cómo generan estados psicológicos críticos en las personas (EPC) y condicionan los resultados del trabajo re-

* La actual investigación se enmarca en un proyecto global sobre estructuras organizacionales laborales formales e informales y en cómo éstas afectan a los valores humanos generales, laborales y organizacionales, a los comportamientos y a las relaciones sociales, dentro y fuera del trabajo. La fase inicial fue el trabajo de tesis doctoral de Genoud en Argentina (2005-2007), anclada en un enfoque cualitativo sobre 33 entrevistas semiestructuradas; luego se avanzó a través de un proceso de triangulación en la Ciudad de Buenos Aires (CABA) y Gran Buenos Aires (GBA), Argentina, incluyendo trabajadores de empresas productivas, servuctivas y logísticas, pertenecientes a todos los niveles jerárquicos.

Tras operacionalizar la guía de entrevista, se construyó un cuestionario que se trabajó en una primera fase 2009-2010 sobre una muestra no paramétrica de 229 casos. Posteriormente se mejoró en una segunda fase 2010-2011, con base en una muestra altamente representativa de la población en estudio (PEA), tras efectuar un muestreo estratificado proporcional con control de cuotas en las variables más relevantes de las que se disponía de información poblacional: actividad y tamaño de la empresa (Genoud, Broveglio y Picasso, 2014). Durante los años 2014-2016 se desarrolló una herramienta de diagnóstico para enriquecer las funciones laborales con base en los datos brindados por 350 trabajadores de países de América Latina. El funcionamiento de dicho modelo fue analizado aplicando modelos de Ecuaciones estructurales (SEM).

1 Gracia, F. J.; Martín, P.; Rodríguez, I. y Peiró, J. M. (2001).

2 MOW (Meaning of Work) es una investigación sobre grupos de trabajadores de distintas categorías, realizada por científicos sociales de 8 países, que diseñaron una encuesta para obtener resultados comparables a nivel europeo con miras a advertir las diferencias del significado del trabajo entre los distintos países entre 1987 y 2002. El tamaño total muestral fue de 5933 individuos. MOW (1987), p. 336.

3 Burke, R. J. (2002); Tang, T. L.; Furnham, A. \& Davis, G. M. (2002). 
alizado (RT), en términos de comportamiento, actitudes y bienestar para la salud del trabajador ${ }^{4}$.

Hay dos razones por las cuales se redobla el esfuerzo por ajustar una herramienta de diagnóstico en América Latina: en primer término, pese a la paradójica forma en que los seres humanos lo experimentan en cada contexto (amor, odio, seguridad, estrés, crecimiento, frustración, etc.) está claro que la modernidad consolidó una sociedad centrada en el trabajo a lo largo de la historia ${ }^{5}$ : el trabajo es un rasgo humano central en la estructuración de la vida personal y la sociedad. El punto es que en los países de la región se han modificado los perfiles exigidos pero las condiciones estructurales ${ }^{6}$ no facilitan la adaptación de la masa trabajadora. La Comisión Económica para América Latina y el Caribe (CEPAL) y la Organización Internacional del Trabajo (OIT) expresan:

"La falta de oportunidades de trabajo decente no solo afecta los ingresos y el bienestar de las personas, sino que también es uno de los factores que "producen una erosión del contrato social básico subyacente en las sociedades democráticas: el derecho de todos a compartir el progreso"

En este sentido, no se trata de replicar teorías o de utilizar modelos importados de otras coyunturas, por el contrario tras 10 años de investigación en el estudio de las dinámicas organizacionales en Argentina y otros países de la región, se busca adaptar la herramienta de diagnóstico, para poder efectivizar la gestión de mejores ambientes laborales.

La segunda razón se funda en la firme convicción de combatir la idea que los seres humanos deberían insistir en liberarse del trabajo, aprovechando ahora las virtudes de la cuarta revolución industrial ${ }^{8}$. Lejos de experimentarse que se asiste a un momento de liberación, los seres humanos están expuestos a desafíos contextuales de reconversión de manera más individual, aunque, como se anticipó, en estas regiones, las oportunidades de acceso no suelen ser

4 Grant, A.M.; Fried, Y. y Juillerat, T. (2010); Morgeson, F. P. y Humphrey, S. E. (2008); Brook, J. B. (1989); Harpaz, I. y Fu, X. (2002); Harpaz, I.; Honig, B. y Coetsier, P. (2002); Parry, J. (2003); Sullivan, P. M. (2003).

5 Agulló Tomás, E. y Ovejero Bernal, A. (2001).

6 La CEPAL y la OIT expresan que la expansión del trabajo por cuenta propia, el surgimiento de nuevas formas de trabajo y una mayor informalidad del empleo asalariado, amenazan el logro del Objetivo de Desarrollo Sostenible $\mathrm{n}^{\circ} 8$ de la Agenda 2030, que busca promover el crecimiento económico sostenido, inclusivo y sostenible, el empleo pleno y productivo y el trabajo decente para todos.

7 Cepal y OIT (2019), p. 5.

8 De la Garza Toledo, E. y Neffa, J. C. (2001). 
justas para todos. La cuestión no excluye a quienes ocupan cargos directivos centrales: en los últimos años, el estudio de la deslocalización y el significado del trabajo en las personas se ha transformado en un tema de actualidad no sólo para el management sino para otras disciplinas sociales ${ }^{9}$. Sin duda, la tecnología y la globalización actual, obligan a los trabajadores a cuestionarse cómo reformular sus perfiles laborales, cómo redoblar los esfuerzos para lograr insertarse en una división internacional del trabajo que alienta la configuración de encadenamientos productivos a través de mecanismos diversos: subcontratación laboral, contratación por proyectos, free lance, olas de emprendedurismo... trabajo flexible. En paralelo, internamente las organizaciones promueven el trabajo en equipo, grupos virtuales, mientras se alientan prácticas de teletrabajo o metodologías ágiles ${ }^{10}$. Quienes gestionan personas deben equilibrar la productividad, adoptando formas de trabajo cuyos orígenes filosóficos se remontan al mundo oriental, pero haciendo foco en el logro de objetivos, la creatividad y no pocas veces la competencia interna entre sus miembros... formas de trabajo que no siempre redundan en eficiencia y eficacia para todos, sino en rotación, ausentismos, pérdida de satisfacción respecto al trabajo, sensación de no crecimiento, insatisfacción con los jefes o intragrupo, sobrecarga, estrés, aburrimiento, en síntesis, pérdida de bienestar.

El objetivo general de este artículo es describir los resultados del trabajo, a partir de los datos relevados mediante una Herramienta de Diagnóstico para Enriquecer las Funciones Laborales (HDEFL), adaptada en América Latina ${ }^{11}$.

En términos específicos, se busca:

- Analizar qué características de diseño de la función laboral, CDT (motivacionales, sociales y contextuales) se correlacionan con los resultados del trabajo (RT), en términos de: resultados de comportamiento (ausentismo y rotación de trabajo), resultados actitudinales (grado de satisfacción en el trabajo, de la necesidad de autocrecimiento, con los superiores, con los compañeros y respecto de la motivación interna) y resultados de bienestar (discriminando sentimientos y factores de presión);

9 Gaggiotti, H. (2004). Gaggiotti indaga la relación que existe entre la deslocalización productiva y el significado del trabajo desde la óptica directiva y presenta el punto de partida de una línea de investigación acerca de cuánto de esta actitud puede forzar a muchas empresas a la deslocalización. El investigador propone que saber qué se entiende por "trabajar" entre los directivos de una empresa puede sugerir la toma de decisiones a la hora de decidir mudar o no una empresa.

10 Wingu (2016).

11 Genoud, M. A.; Broveglio, G. D. y Picasso, E. (2018). 
- Indagar el rol de los estados psicológicos críticos (EPC) como mediadores entre los CDT y los RT. En este sentido primero se busca determinar cuáles son las características motivacionales, sociales y contextuales de la función laboral, que impactan en el grado de significatividad atribuido al trabajo, el grado de responsabilidad asumido y el de conocimientos de los resultados alcanzados. A posteriori, se analiza cuál de los EPC tiene mayor peso relativo en los RT y se describe comparativamente si las CDT o los EPC tienen el mismo impacto en los RT descritos por los trabajadores.

El documento se estructura en términos de 4 secciones. El apartado I presenta la herramienta HDEFL, sintetizando los aspectos teóricos desde los cuales se ancla la interpretación de los resultados que se describirán. La sección II expone el camino metodológico transitado para concretar los objetivos de la investigación global y las características de la muestra. La parte III describe el análisis de los datos obtenidos vía el cuestionario que operacionalizó la herramienta adaptada. Finalmente, el apartado IV plantea una síntesis en términos de RT con miras a proponer algunas sugerencias, se explicitan las limitaciones del artículo y se plantean los futuros análisis que se podrán presentar utilizando la herramienta.

\section{LA HERRAMIENTA HDEFL}

La herramienta de diagnóstico del Diseño del trabajo, HDEFL ${ }^{12}$ ha sido adaptada del Modelo de las características del puesto (MCP) de Hackman y Oldham en sus diversas revisiones (1974, 1975, 1976, 1980); Kulik y Oldham (1988), del modelo de trabajo ampliado de Morgeson y Humphrey (2006, 2008) y Humphrey, Morgeson y Nahrgang (2007), Parker, Morgeson y Johns (2017) y con base en la investigación sobre estructuras laborales realizada durante 10 años en Argentina por Genoud, Broveglio y Picasso ${ }^{13}$. En este sentido se alinea a la perspectiva de Griffin (1982), quien advirtió que los investigadores y quienes gestionan, deben tener cuidado de no dejar de buscar mejores y más precisas formulaciones para el diseño del trabajo, así como evaluar continuamente las formulaciones actuales.

12 Genoud, M. A.; Broveglio, G. y Picasso, E. (2018). El trabajo permite profundizar el diseño de la herramienta y diagnosticar la significatividad, responsabilidad y conocimiento de los resultados laborales mediante modelos de ecuaciones estructurales.

13 Genoud, M. A.; Broveglio, G. y Picasso, E. (2014, 2018). 
La construcción de la herramienta amplía la manera de resignificar el trabajo, asumiendo que hoy los resultados de las funciones laborales deben analizarse integrando nuevas características estructurales del diseño del trabajo, incluyendo diversas especificaciones de quienes las llevan a cabo, así como variables ${ }^{14}$ que hacen posible evaluar la dinámica del trabajo en equipo ${ }^{15}$, abordando por ejemplo tareas, objetivos, roles de cada miembro, soportes organizacionales y enlaces con el contexto organizacional más amplio. Específicamente, el instrumento integra cuatro perspectivas diferentes para el diseño del trabajo: por un lado la motivacional (profundizada luego por Hackman, J. R. y Lawler, E. E. (1971) y Hackman, J. R. y Oldham, G. R. (1975); anclada en el enfoque de la psicología organizacional y enfocada en la maximización de la satisfacción laboral, la retención y la motivación interna); se suma la perspectiva mecanicista (enraizada en la gestión científica y el enfoque de la ingeniería industrial, con foco en la maximización de la eficiencia); la perspectiva biológica (anclada en la ergonomía y las ciencias médicas que hace hincapié en el trabajo de diseño para maximizar el confort y la salud física) y el modelo perceptivo-motriz de la psicología experimental y factores humanos, que considera la atención y demandas informativas del trabajo ${ }^{16}$.

La herramienta pone énfasis en diagnosticar funciones o roles laborales, más que en diseñar puestos de trabajo ${ }^{17}$ : en este sentido permite considerar no solo las tareas y sus demandas específicas, sino aspectos sociales y contextuales; asimismo otorga flexibilidad, porque da importancia a cuestiones formales pero también permite considerar elementos idiosincráticos, discrecionales o emergentes; finalmente permite reconocer que las personas no siempre se ajustan a determinados roles, de manera que facilita la elección de la composición de las unidades de trabajo. La figura $\mathrm{n}^{\circ} 1$ sintetiza la herramienta.

14 Kozlowski, S. W. J. y Ilgen, D. R. (2006).

15 Campion, M. A.; Medsker, G. J., Higgs, A. C. (1993); Guzzo, R. A. y Dickson, M. W. (1996); Hackman, J. R. (1987); Perretti, F. y Negro, G. (2006); Stewart, G. L. (2006); Morgeson, F. P. y Humphrey, S. E. (2008).

16 Campion, M. A. (1988); Campion M. A. y Thayer P. W. (1985); Grant, A. M.; Fried, Y. \& Juillerat, T. (2010).

17 Davis, L. E. y Taylor, J. C. (1979). 
Figura $n^{0} 1$ : Herramienta de Diagnostico para el enriquecimiento de las Funciones Labores (HDEFL)

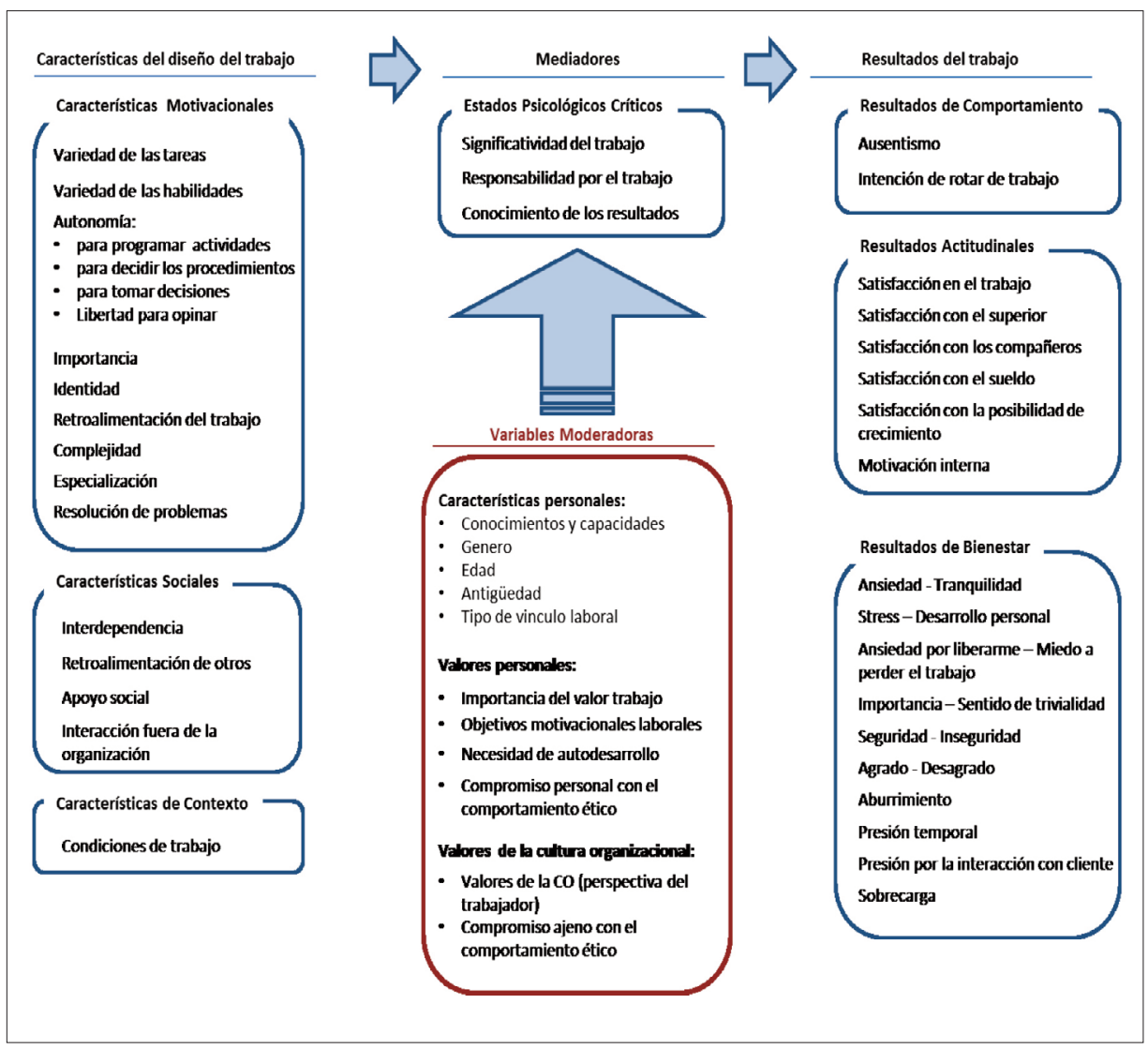

Fuente: Herramienta ampliada por Genoud M. A.; Broveglio, G. y Picasso, E. (2018).

La HDEFL articula la relación entre las CDT, los EPC y los RT; asimismo posibilita analizar la injerencia de variables moderadoras (VM).

Las CDT incluyen las características motivacionales, sociales y contextuales. Las motivacionales ${ }^{18}$ permiten profundizar el grado de variedad de las

18 Humphrey, S. E..; Nahrgang, J. D. y Morgeson, F. P. (2007) resaltan que la variedad de tareas y habilidades no se separan como constructos distintos ni en el MCP (1976), ni en el fob Characteristics Inventory de Sims, H. P.; Szilagyi, A. D. y Keller, R. T. (1976), tampoco Fried, Y. y Ferris, G. R. (1987). Asimismo, la autonomía ha sido un concepto que en el MCP (1976) fue analizado como un constructo unidimensional, pero Breaugh J. A. (1985); Morgeson, F. P. y Humphrey, S. E. (2006) y Jackson, P. R.; Wall, T. D.; Martin, R. y Davids, K. (1993) han sugerido distintas facetas. 
tareas (como se anticipó, con base en el trabajo del equipo sobre estructuras laborales, se replicó un diferencial semántico para profundizar el alcance que posee el puesto de trabajo, en términos de repetitividad, monotonía, complejidad y tipo de objetivos); la variedad de habilidades (tipo de destrezas y habilidades que retan el desempeño); la autonomía (ahondando cuanta libertad se posee para programar actividades, decidir procedimientos, tomar decisiones u opinar); la importancia (grado en el cual los trabajadores experimentan que el trabajo tiene un impacto perceptible y sustancial para el trabajador en la vida de otras personas, en la organización o en la sociedad); la identidad (indaga en qué medida cada trabajador experimenta que puede realizar una tarea de principio a fin, permitiendo o no apreciar su aporte en el producto final); la retroalimentación del trabajo (que alude al tipo de feedback que experimenta el trabajador, diferenciando aspectos que tienen que ver con la posibilidad de autoevaluarse, corregirse y el grado en que trabajador recibe información clara y directa de otros, sobre los resultados del trabajo); la especialización (grado en que el puesto demanda calificación o profesionalidad) y el grado en que el trabajo ofrece oportunidad de resolución de los problemas (conceptualmente similar al grado de creatividad) ${ }^{19}$. Las características sociales abarcan el análisis de la interdependencia (grado en que un trabajo es contingente con la labor de otros); la retroalimentación interpersonal, el apoyo social y la interacción fuera de la organización (con clientes, proveedores, etc.) ${ }^{20}$. Finalmente, en el marco de las características contextuales se abordan las condiciones de trabajo $^{21}$.

Como se anticipó, Hackman y Oldham (1976) plantearon que las Características Motivacionales impactaban en los resultados mediando tres Estados Psicológicos Críticos (EPC): la significatividad del trabajo (el grado de sentido y de significación atribuida al trabajo), la responsabilidad por el trabajo realizado (es decir, el grado en que un empleado se siente responsable de rendir cuentas por los resultados de trabajo) y el grado de conocimiento de los resultados de las actividades realizadas (es decir, el grado en que el empleado es consciente de su nivel de rendimiento).

Los Resultados del Trabajo se evalúan en tres dimensiones: los de comportamiento (se abordan dos indicadores: el ausentismo y la intención de ro-

19 Edwards, J. R.; Scully, J. A. y Brtek, M. D. (2000); Karasek, R. A. (1998).

20 Parker, S. K., y Wall, T. D. (2001); Ilgen, D. R. y Hollenbeck, J. R. (1991).

21 Campion, M. A. y Thayer, P. W. (1985); Humphrey S. E. Nahrgang, J. D. y Morgeson, F. P. (2007). 
tación); los actitudinales (evaluados a través del grado de satisfacción en el trabajo, con el supervisor, con los compañeros, con el sueldo y con la posibilidad de autocrecimiento y el grado de motivación interna); por último, los resultados de bienestar, a través de los sentimientos verbalizados (evaluados a través de un diferencial semántico que aborda estados de ansiedad, estrés, miedo a perder el trabajo, sentido de trivialidad, seguridad y agrado) y factores de presión (el tiempo, la sobrecarga, el grado de interacción con el cliente, el aburrimiento $)^{22}$.

Finalmente resta precisar que el modelo incluye variables moderadoras (VM). En este sentido, la herramienta permite segmentar la información por variables sociodemográficas, como nivel educativo, género ${ }^{23}$, edad, antigüedad en la empresa y tipo de vínculo laboral. Asimismo, se han incluido preguntas que abordan la descripción de los valores laborales personales y organizacionales; se asume que los seres humanos forjan prioridades de valores humanos básicos entre los cuales el trabajo suele ocupar un sitio importante, aunque varíe su significación relativa a medida que se enfrentan distintas experiencias coyunturales ${ }^{24}$. Los seres humanos poseen construcciones motivacionales generales y laborales, ambas se estructuran de manera circular, dinámica y continua, evidencian un patrón total de relaciones de conflicto y congruencia que suelen solaparse, se refieren a objetivos deseables que los individuos se esfuerzan por obtener, razón por la cual guían la selección o evaluación de acciones, políticas, personas, relaciones y eventos. Entender las expectativas relativas a prioridades laborales, asociarlas a las características del trabajo que se realiza, confrontarlas con los valores que la organización promueve desde la mirada de los actores, facilita el diagnóstico de propuestas de gestión para mejorar los resultados del trabajo.

\section{EL CAMINO METODOLÓGICO}

La tipología del estudio es exploratoria, descriptiva y explicativa. Desde el punto de vista teórico-metodológico, se busca exacerbar cortes interpretativos transdisciplinarios en los estudios organizacionales ${ }^{25}$, anclados en la So-

22 Genoud M. A.; Broveglio, G. y Picasso, E. (2014).

23 García-Bernal, J.; Gargallo-Castel, A.; Marzo-Navarro, M. y Rivera-Torres, P. (2005).

24 Drenth, P. J. D. (1991); Schwartz, S. H. (1992, 2005a, 2005b); Ros, M.; Schwartz, S. H. \& Surkiss, S. (1999); Genoud, M. A. (2009); Genoud M. A.; Broveglio, G. y Picasso, E. (2012); Gracia, F. J. Martin, P.; Rodríguez, I. y Peiró, J.M. (2001).

25 Pérez, A. y Guzmán, M. (2015). 
ciología del Trabajo, las ciencias de la Administración y la Psicología Organizacional; la idea es sumar aportes a la corriente de Nuevos Estudios laborales en América Latina (NEL) en línea con De la Garza Toledo (2000).

El muestreo no probabilístico incluye sujetos voluntarios que respondieron un cuestionario disponible online durante 2016; la muestra abarca 423 trabajadores latinoamericanos, con mayoría de Argentina y Brasil. En síntesis, se trata de una muestra con un alto grado de instrucción mayoritariamente universitario incompleto y con una distribución jerárquica claramente piramidal. En términos generacionales predomina la generación entre 22 y 35 años por sobre el resto, tratándose de trabajadores pertenecientes a empresas grandes en un $48,94 \%$, medianas en un $14,18 \%$, pequeñas en un $7,80 \%$ y micro en un 29,08\%. Las tablas $n^{\circ} 1,2,34$ y 5 amplían la información.

Tabla 1: Configuración muestral de origen según tipo de actividad principal de la empresa

\begin{tabular}{l|c|c}
\hline Tipo de actividad principal de la empresa & $\begin{array}{c}\text { Frecuencias } \\
\text { absolutas }\end{array}$ & $\begin{array}{c}\text { Frecuencias } \\
\text { relativas }\end{array}$ \\
\hline $\begin{array}{l}\text { Productiva (Ejemplos.: Industria, } \\
\text { laboratorio, construcción, etc.) }\end{array}$ & 115 & $27,19 \%$ \\
\hline $\begin{array}{l}\text { De servicios, logística o comercial } \\
\text { (Ejemplos. Comercios, importadores, } \\
\text { hipermercados, Bancos, seguros, } \\
\text { tecnología, comunicaciones, etc.) }\end{array}$ & 308 & $72,81 \%$ \\
\hline \begin{tabular}{l} 
Muestra \\
\hline
\end{tabular}
\end{tabular}

Fuente: elaboración de los autores. 
EL TRABAJO COMO FUENTE DE RESULTADOS. HACIA UNA CONCEPTUALIZACIÓN MÁS

INTEGRADA DEL DIAGNÓSTICO DEL ENRIQUECIMIENTO DEL TRABAJO EN AMÉRICA LATINA

Tabla 2: Reagrupamiento de las categorías de los niveles educativos

\section{Grado de nivel educativo alcanzado}

Frecuencias relativas

Reagrupamiento

\begin{tabular}{lll}
\hline Posgrado & $23,64 \%$ & Alto \\
\hline Universitario completo & $13,71 \%$ & \\
\hline Terciario completo & $5,44 \%$ & \\
\hline Universitario incompleto & $44,21 \%$ & Medio \\
\hline Terciario incompleto & $3,55 \%$ & \\
\hline Secundario completo & $8,98 \%$ & \\
\hline Secundario incompleto & $0,47 \%$ & Bajo \\
\hline Primario completo & $0,00 \%$ & \\
\hline Primario incompleto & $0,00 \%$ & \\
\hline
\end{tabular}

Fuente: elaboración de los autores.

Tabla 3: Configuración muestral de origen según nivel jerárquico descripto por los encuestados

Nivel jerárquico descripto por el encuestado

Frecuencias relativas

\begin{tabular}{lc} 
Dueño & $10,17 \%$ \\
\hline Gerente General/Director & $4,73 \%$ \\
\hline Gerente (nivel alto) & $5,91 \%$ \\
\hline Jefe, supervisor (nivel intermedio) & $23,17 \%$ \\
\hline Colaborador- sin personas a cargo (operario, analista, etc.) & $56,02 \%$ \\
\hline Muestra = 423 & $100,00 \%$ \\
\hline
\end{tabular}

Fuente: elaboración de los autores.

Tabla 4: Distribución por edad según generación

\begin{tabular}{|c|c|c|}
\hline Rango & Frecuencias absolutas & Frecuencias relativas \\
\hline $\begin{array}{c}1981-1994 \\
22-35\end{array}$ & 271 & $64,07 \%$ \\
\hline $\begin{array}{c}1965-1980 \\
36-54\end{array}$ & 110 & $26,00 \%$ \\
\hline $\begin{array}{c}1945-1964 \\
55-71 \\
\end{array}$ & 42 & $9,93 \%$ \\
\hline
\end{tabular}

Fuente: elaboración de los autores. 
Tabla 5: Configuración porcentual muestral de las empresas según su tamaño

Tamaño de las empresas según su dotación de personal Frecuencias relativas

\begin{tabular}{lc}
\hline Grandes (> 300 empleados) & $48,94 \%$ \\
\hline Medianas (101 a 299 empleados) & $14,18 \%$ \\
\hline Pequeñas (51 a 100 empleados) & $7,80 \%$ \\
\hline Micro (<50 empleados) & $29,08 \%$ \\
\hline Muestra: 423 & $100,00 \%$ \\
\hline
\end{tabular}

Fuente: elaboración de los autores.

El cuestionario construido contempló la evaluación de las preguntas del MCP o fob Design Survey (JDS) de Hackman y Oldham, en sus diversas versiones ${ }^{26}$, si bien incluyó algunos interrogantes idénticos, otros se formularon de manera diferente así como se incorporaron nuevas preguntas con base en el aprendizaje capitalizado sobre el estudio de estructuras laborales, realizado desde el año 2009 por los autores. La idea fue enriquecer el instrumento de medición reflejando otras variables a considerar en América Latina, como por ejemplo el tema valores, sentimientos y factores de presión.

Como primer procedimiento de análisis se aplicó la metodología de correlación canónica para explorar y determinar aquellas variables más relevantes dentro de cada grupo de variables que conforman el modelo estudiado: los CDT, los EPC y los RT.

Las correlaciones canónicas se utilizan cuando un conjunto de variables multivariantes puede dividirse en dos grupos homogéneos y se desea estudiar la relación entre ambos conjuntos de variables. El análisis se focaliza en la correlación entre una combinación lineal de variables de un determinado grupo y una combinación lineal de variables de otro grupo. Estas combinaciones lineales determinan nuevas variables ${ }^{27}$. El objetivo principal es determinar el par de combinaciones lineales que tienen la mayor correlación, las cuales representan la primera dimensión extraída. Luego, se determina otro par de

26 En concordancia con Fortea, M.; Fuertes, F. y Agost, R. (1994), numerosos autores plantean los problemas de medición del instrumento (Ilgen, D. R. y Hollenbeck, J. R. (1991)), así como otros argumentan que, probablemente, algunas de las características del puesto correlacionan en la realidad. Hackman, J. R. y Oldham, G. R. (1974); Birnbaum, P.; Farh, J. \& Wong, G. (1986); Kulik, C. T. y Oldham, G. R. (1988), entre otros.

27 Peña, D. (2013). 
combinaciones lineales con la siguiente mayor correlación, pero cuyas combinaciones lineales no están correlacionadas con las primeras, representando a la segunda dimensión. De esta misma manera se construyen las dimensiones restantes. Los pares de combinaciones lineales se denominar variables canónicas, y sus correlaciones, correlaciones canónicas. El método construye tantas variables canónicas como el mínimo de variables de los dos grupos ${ }^{28}$.

Para este análisis, se extrajeron solamente las dos primeras dimensiones, dado que la pérdida del ajuste no fue superior a 0,6 , lo que significa que aproximadamente el $70 \%$ de la variabilidad conjunta fue explicada por estas primeras dos dimensiones, para todos los grupos relacionados. Este método permitió reducir el número de variables y extraer solamente aquellas que se consideraron más importantes al estudio. Esto significa extraer las variables de cada grupo que tuvieran los mayores aportes a cada dimensión, siendo las variables más relevantes.

A posteriori, el segundo análisis fue medir las relaciones entre las variables extraídas de cada grupo mediante el cálculo del coeficiente de correlación de rangos de Spearman ${ }^{29}$, respetando las formulaciones teóricas del modelo. De esta manera se pudo observar y determinar el grado de relación entre pares de variables, ya sea por relacionarse de manera directa o inversa: la relación directa se dio en el caso de aquellas preguntas cuyas respuestas poseían el mismo signo, y la relación inversa cuando una de las respuestas era de signo distinto a la otra.

Cabe aclarar que en las tablas del apartado siguiente, se observarán signos positivos y negativos como resultado de cada comparación. Dos ejemplos de lo expuesto anteriormente se visualizan al comparar las preguntas 7 y 15 . En el caso específico de la pregunta 7, por tratarse de un diferencial semántico elaborado para evaluar el grado de especialización de las funciones laborales, el encuestado debía calificar entre dos conjuntos opuestos de factores o adjetivos bipolares ${ }^{30}$, orientándose con una puntuación mayor cuando el trabajo era altamente especializado (tareas repetitivas, simples y monótonas, con objetivos de alto nivel de detalle) versus hacia una puntuación menor cuando se trataba de un trabajo poco especializado (tareas no repetitivas, complejas, diversas y con objetivos de bajo nivel de detalle). Respecto a la pregunta 15,

28 Johnson, R. \& Wichern, D. (2001).

29 El coeficiente de correlación de Spearman es un coeficiente que puede ser utilizado para medir la relación entre dos variables ordinales, por lo que es apropiado para este estudio. Conover, W. J. (1980).

30 Hernández Sampieri, R.; Fernandez Collado, C. y Baptista Lucio, P. (2001). 
se incluyeron afirmaciones con direcciones positivas que evidenciaban que el encuestado calificaba favorablemente sus resultados actitudinales frente a la labor desempeñada (discriminando el grado de satisfacción en el trabajo (15.4), con el supervisor (15.7), con los compañeros (15.8), con el sueldo (15.9) y con la posibilidad de autocrecimiento (15.6)) y otra afirmación negativa con sentido inverso (el grado de motivación interna (15.5)), para contrarrestar el sesgo de respuesta automática en la categoría de opción intermedia ${ }^{31}$ o la opción de respuesta afirmativa (aquiescencia) ${ }^{32}$.

Resta precisar que en el apartado siguiente, el análisis destacará aquellas CDT que más impactan en los RT o en los EPC, aunque el lector podrá ampliar la información disponible en las tablas. El mismo criterio se utiliza cuando se analiza la incidencia de los EPC en los RT.

\section{LO QUE LAS PERSONAS REVELAN ACERCA DE LOS RESULTADOS DEL TRABAJO.}

El modelo desarrollado permite identificar qué CDT y qué EPC podrían estimular mejores RT en los trabajadores.

En línea con los objetivos específicos planteados:

3.1 Se describen qué características motivacionales, sociales y contextuales de la función laboral se correlacionan con los resultados de comportamiento (ausentismo y rotación de trabajo), actitudinales (satisfacción en el trabajo, con el autocrecimiento, con los superiores, con los compañeros y motivación interna) y de bienestar (discriminando sentimientos y factores de presión);

3.2 Se especifica qué $E P C$ tiene mayor peso relativo en los $R T$; en este sentido se busca determinar qué $C D T$ los estimulan (motivacionales, sociales y/o contextuales) y cómo estos EPC, median los resultados.

3.1 Las Características del Diseño de la función laboral que estimulan los Resultados del Trabajo.

31 Díaz de Rada, V. (2001).

32 Morales, P. (2006). 
A continuación, se describen los RT en términos de tres dimensiones: indicadores de comportamiento; de actitudes y de bienestar.

¿Qué Características de Diseño de la función laboral (CDT) deberían considerarse, si se desean mejorar los Resultados de Comportamiento (RC), en términos de ausentismo e intención de rotar?

Los trabajadores expresan que la posibilidad de ser creativos y la amplitud comunicacional respecto a manifestar lo que molesta o preocupa, así como recibir información clara y directa de sus resultados, atenúa el deseo de ausentarse y la intención de rotar. Es válido resaltar que experimentar condiciones de trabajo buenas en términos de seguridad, atenúa la intención de rotar. La Tabla nº amplía la información.

Tabla n 6: Características de Diseño de la función laboral (CDT) y Resultados de Comportamiento (RC)

\begin{tabular}{l|l|c|}
\cline { 2 - 3 } \multicolumn{1}{l|}{} & \multicolumn{2}{|c|}{$\begin{array}{c}\text { Resultados de Comportamiento (RC) } \\
\text { (correlaciones de Spearman) }\end{array}$} \\
\hline $\begin{array}{l}\text { Características de Diseño de la función la- } \\
\text { boral (CDT): Motivacionales (CM), Sociales } \\
\text { (CS) y Contextuales (CC) }\end{array}$ & $\begin{array}{l}\text { Ausentismo } \\
\text { (P. 11.7) }\end{array}$ & $\begin{array}{c}\text { Intención de rotar en } \\
\text { el trabajo (P. 11.6) }\end{array}$ \\
\hline $\begin{array}{l}\text { Poder ser creativo (Resolución de proble- } \\
\text { mas, P. 11.2; CM) }\end{array}$ & $-0,303$ & $-0,162$ \\
\hline $\begin{array}{l}\text { Autonomía en términos de libertad de ex- } \\
\text { presar lo que molesta, lo que preocupa (P. } \\
\text { 19.1; CM) }\end{array}$ & $-0,300$ & $-0,279$ \\
\hline $\begin{array}{l}\text { Recibir información clara y directa sobre } \\
\text { los Resultados del Trabajo (Retroalimen- } \\
\text { tación del trabajo, P. 11.3; CM y S) }\end{array}$ & $-0,256$ & $-0,251$ \\
\hline $\begin{array}{l}\text { Condiciones de trabajo: físicas, comodida- } \\
\text { des, seguridad (P. 15.10; CC) }\end{array}$ & $-0,277$ & $-0,346$ \\
\hline
\end{tabular}

Fuente: elaboración de los autores. 
- ¿Qué Características de Diseño de la función laboral (CDT) estimulan los Resultados Actitudinales (RA), en pos de lograr mayor grado de satisfacción y motivación interna en el trabajo?

En términos generales, se observa que diversas características motivacionales $(\mathrm{CM})$, sociales (CS) y contextuales (CC) de la función laboral, estimulan distintos grados de satisfacción, aunque no es tan sencillo activar la motivación interna.

En primer término, recibir información clara y directa sobre los resultados del trabajo esta correlacionado de manera diversa con los resultados actitudinales: favorece el nivel de satisfacción en el trabajo $(0,412)$ y con el superior $(0,406)^{33}$, asimismo estimula la posibilidad de crecer $(0,326)$, equilibra la percepción de satisfacción respecto al sueldo $(0,299)$ y el vínculo con los compañeros $(0,305)$; en este sentido cabe resaltar que no es la consulta con los pares, lo que mejora la satisfacción interpersonal $(0,146)$. Este factor también activa la motivación interna $(-0,288)$.

En segundo lugar, la posibilidad de ser creativos y fundamentalmente de ser autónomos $^{34}$ (porque se puede tomar decisiones con base en la experiencia o en los conocimientos disponibles; se puede opinar o se puede influir en los objetivos propios), activan el grado de satisfacción del trabajo y de quienes buscan crecer en su función; cuando se gana autonomía por disponer de libertad para influir sobre la definición de los propios objetivos, se incrementa la motivación interna $(-0,297)$.

El grado de importancia que cree el trabajador que tiene el puesto en la organización, mejora la posibilidad de crecimiento $(0,364)$, mejora la satisfacción del trabajo $(0,343)$ y la relación con su superior $(-0,29)$, así como eleva el nivel de motivación interna $(-0,290)^{35}$.

33 La investigación coincide con los estudios realizados en el meta análisis de Humphrey, S. E.; Nahrgang, J. D. y Morgeson, F. P. (2007) en términos de resultados actitudinales y bienestar (en relación a la ansiedad), sin embargo, los autores no señalan correlación en términos de resultados de comportamiento.

34 Como se anticipó, la autonomía requiere analizar múltiples facetas. En concordancia con Morgeson F. P. y Humphrey, S.E. (2008) particularmente la posibilidad de tomar decisiones tiene un alto impacto en la satisfacción con el trabajo, con los compañeros, así como alienta la motivación interna, contribuye a bajar el ausentismo y mejora el bienestar (bajando el stress y la ansiedad).

35 Los estudios de Grant A. M. y Morgeson F. P. coinciden en que si los trabajadores sienten que su tarea es importante mejora la satisfacción del trabajo y la motivación; como se verá adicionalmente la investigación evidencia que se activan los tres EPC simultáneamente. 
Cabe señalar que también el sentido de identidad que percibe el trabajador parece promover una mejor relación con su jefe y es lo que más se correlaciona con la motivación interna (ambas en un 0,329 ), en concordancia con Morgeson, F.P. y Humphrey, S.E. (2008).

Es válido destacar que la variedad de tareas- tanto en relación con la diversidad que poseen o a su grado de repetitividad- estimulan la satisfacción respecto a la posibilidad de crecimiento $(0,384)$ y con el trabajo $(0,325)^{36}$.

En relación con las condiciones contextuales, ellas mejoran la satisfacción con el jefe $(0,401)$, el trabajo $(0,334)$ y con los compañeros $(0,324)$ y es el único factor de las características de diseño (CDT) que contribuye a equilibrar la perspectiva respecto a la remuneración $(0,468)$.

Los datos evidencian que parece muy difícil activar la satisfacción con los compañeros de trabajo, realidad preocupante en un contexto laboral en el que el trabajo equipo es una tendencia cada vez más necesaria. La tabla $\mathrm{n}^{\circ} 7$ amplía la información.

36 Morgeson F. P. y Humphrey, S.E. (2008) coinciden en esta correlación $(0,46)$, pero también indican que debe observarse si no se genera sobrecarga. 
Tabla $n^{0}$ 7: Características de Diseño de la función laboral (CDT): y Resultados actitudinales (RA) (Grados de Satisfacción y Motivación Interna)

\begin{tabular}{|c|c|c|c|c|c|c|}
\hline & Res & tos acti & nales (RA) & Correlacio & nes de Spea & arman) \\
\hline $\begin{array}{l}\text { Características de } \\
\text { Diseño de la fun- } \\
\text { ción laboral } \\
\text { (CDT): Motivacio- } \\
\text { nal (CM), Social } \\
\text { (CS) y Contextual } \\
\text { (CC) }\end{array}$ & $\begin{array}{l}\text { Satisf. con } \\
\text { la posib. de } \\
\text { crecimiento } \\
\text { (P.15.6) }\end{array}$ & $\begin{array}{l}\text { Satisf. del } \\
\text { trabajo } \\
\text { (P.15.4) }\end{array}$ & \begin{tabular}{|c|} 
Satisf. con \\
el superior \\
(P. 15.7)
\end{tabular} & $\begin{array}{l}\text { Satisf. con } \\
\text { los comp. } \\
\text { (P.15.8) }\end{array}$ & $\begin{array}{l}\text { Satisf. con } \\
\text { el sueldo } \\
\text { (P.15.9) }\end{array}$ & $\begin{array}{c}\text { Motivación } \\
\text { interna } \\
\text { (P.15.5) }\end{array}$ \\
\hline $\begin{array}{l}\text { Variedad de ta- } \\
\text { reas: diversidad } \\
\text { versus monotonía } \\
\text { (P. } 7.3 \text { ) }\end{array}$ & $-0,384$ & $-0,325$ & 0,217 & $-0,093$ & $-0,049$ & 0,217 \\
\hline $\begin{array}{l}\text { Variedad de ta- } \\
\text { reas: no repetitivas } \\
\text { versus repetitivas } \\
\text { (p. 7.1) }\end{array}$ & $-0,381$ & $-0,281$ & $-0,131$ & $-0,082$ & $-0,046$ & 0,131 \\
\hline $\begin{array}{l}\text { Poder ser Creativo } \\
\text { (Resolución de } \\
\text { problemas, P. 11.2; } \\
\text { CM) }\end{array}$ & 0,456 & 0,368 & $-0,257$ & 0,130 & 0,080 & $-0,257$ \\
\hline $\begin{array}{l}\text { Autonomía para } \\
\text { tomar decisiones } \\
\text { según experiencia } \\
\text { o conocimientos } \\
(19.4 ; \text { CM) }\end{array}$ & 0,407 & 0,368 & $-0,280$ & 0,179 & 0,161 & 0,179 \\
\hline $\begin{array}{l}\text { Autonomía en tér- } \\
\text { minos de libertad } \\
\text { de opinar (19.1) }\end{array}$ & 0,279 & 0,374 & $-0,261$ & 0,274 & 0,233 & $-0,261$ \\
\hline $\begin{array}{l}\text { Identidad (14.2; } \\
\text { CM) }\end{array}$ & $-0,271$ & $-0,242$ & 0,329 & $-0,101$ & $-0,008$ & 0,329 \\
\hline $\begin{array}{l}\text { Grado de impor- } \\
\text { tancia del puesto } \\
\text { en la organización } \\
(14,4 ; C M)\end{array}$ & 0,364 & 0,343 & $-0,29$ & $-0,030$ & 0,159 & $-0,290$ \\
\hline
\end{tabular}




\begin{tabular}{|c|c|c|c|c|c|c|}
\hline $\begin{array}{l}\text { Libertad para in- } \\
\text { fluir sobre la defi- } \\
\text { nición de mis ob- } \\
\text { jetivos (19.3; CM) }\end{array}$ & 0,388 & 0,333 & $-0,297$ & $-0,148$ & 0,188 & $-0,297$ \\
\hline $\begin{array}{l}\text { Recibir informa- } \\
\text { ción clara y di- } \\
\text { recta sobre los } \\
\text { Resultados del } \\
\text { Trabajo (Retroali- } \\
\text { mentación del } \\
\text { trabajo, P. 11.3; } \\
\text { CMyS) }\end{array}$ & 0,326 & 0,412 & 0,406 & 0,305 & 0,299 & $-0,288$ \\
\hline $\begin{array}{l}\text { El trabajo re- } \\
\text { quiere consultar } \\
\text { información de } \\
\text { otras personas } \\
\text { (Retroalimenta- } \\
\text { ción de otros, } \\
14.7 ; \text { CS) }\end{array}$ & 0,199 & 0,196 & 0,187 & 0,146 & 0,031 & $-0,139$ \\
\hline $\begin{array}{l}\text { Condiciones de } \\
\text { trabajo: físicas, } \\
\text { comodidades, } \\
\text { seguridad, } \\
\text { P.15.10; CC) }\end{array}$ & 0,224 & 0,334 & 0,401 & 0,324 & 0,468 & $-0,25$ \\
\hline
\end{tabular}

Fuente: elaboración de los autores.

- ¿Qué Características de Diseño de la función laboral (CDT) mejoran los Resultados de Bienestar (RB)?

La consideración individual de las características motivacionales, sociales y contextuales no revela correlación significativa contundente, ni con todos los indicadores de sentimientos de bienestar ni con todos los factores de presión. Cabe aclarar que los RB se ahondaron a través de dos preguntas: la P. 17 indagaba sentimientos, a través de un diferencial semántico que permite comprender en qué medida las personas expresan que les agrada ir a trabajar o no; cómo se asocia el tener o no trabajo con la seguridad versus la inseguridad; si el trabajo constituye una fuente de desarrollo o de estrés; si el trabajador expresa grado de ansiedad por liberarse del trabajo o se inclina por padecer miedo a perderlo, si prima la tranquilidad o la ansiedad en la función laboral y si el trabajo realizado vale la pena o se experimenta un sentido trivial. Por 
su parte la P. 16, buscaba identificar factores de presión, como el aburrimiento, la presión temporal, la injerencia de demandas cambiantes e inmediatas (por ej. rigidez de horarios), el grado de interacción con el cliente y el nivel de sobrecarga del trabajo.

Resulta interesante observar que cuando las personas perciben que poseen mayor autonomía para tomar decisiones $(0,356)$, así como también cuando experimentan la posibilidad de sentirse creativos $(0,292)$, independientemente del nivel educativo que posean, y poseen buenas condiciones de trabajo (físicas, seguras, cómodas) (0,273), aumenta el bienestar, porque se opaca el aburrimiento; asimismo estas variables en su conjunto, permiten que se active el trabajo como un lugar al que se acude con agrado, se compense la sensación de estrés en favor del desarrollo, se gane tranquilidad y se atenúe el sentido de trivialidad de las tareas. No hay denuncias de sobrecarga. La tabla $n^{\circ} 8$ sintetiza lo expuesto. 
EL TRABAJO COMO FUENTE DE RESULTADOS. HACIA UNA CONCEPTUALIZACIÓN MÁS INTEGRADA DEL DIAGNÓSTICO DEL ENRIQUECIMIENTO DEL TRABAJO EN AMÉRICA LATINA

Tabla no 8: Características de Diseño de la función laboral (CDT): y Resultados de Bienestar (RB)

\begin{tabular}{|c|c|c|c|c|c|}
\hline & Result & de Bier & (RB) (Co & ación de Sp & rman) \\
\hline $\begin{array}{l}\text { Características de Di- } \\
\text { seño de la función la- } \\
\text { boral (CDT): Caracte- } \\
\text { rísticas } \\
\text { Motivacionales (CM); } \\
\text { Sociales (CS) y Con- } \\
\text { textuales (CS) }\end{array}$ & $\begin{array}{l}\text { Aburri- } \\
\text { miento } \\
(16.1)\end{array}$ & $\begin{array}{c}\text { Grado de } \\
\text { agrado ha- } \\
\text { cia el tra- } \\
\text { bajo que se } \\
\text { realiza- } \\
\text { Grado de } \\
\text { desagrado } \\
\text { (17.1) }\end{array}$ & $\begin{array}{l}\text { Stress - } \\
\text { Desarrollo } \\
\text { personal } \\
(17.3)\end{array}$ & $\begin{array}{c}\text { Ansiedad - } \\
\text { Tranquilidad } \\
(17.5)\end{array}$ & $\begin{array}{l}\text { Siento que } \\
\text { mi trabajo } \\
\text { vale la pena- } \\
\text { Siento que } \\
\text { mi trabajo es } \\
\text { trivial (17.6) }\end{array}$ \\
\hline $\begin{array}{l}\text { Autonomía para to- } \\
\text { mar decisiones según } \\
\text { experiencia o conoci- } \\
\text { mientos (19.4; CM) }\end{array}$ & 0,356 & $-0,336$ & $-0,252$ & 0,177 & $-0,362$ \\
\hline $\begin{array}{l}\text { Autonomía para de- } \\
\text { cidir los procedi- } \\
\text { mientos (11.1) }\end{array}$ & 0,313 & $-0,255$ & $-0,244$ & 0,247 & $-0,244$ \\
\hline $\begin{array}{l}\text { Autonomía en tér- } \\
\text { minos de libertad de } \\
\text { expresar los que } \\
\text { molesta, lo que pre- } \\
\text { ocupa (19.1) }\end{array}$ & 0,271 & $-0,335$ & $-0,244$ & 0,247 & $-0,302$ \\
\hline $\begin{array}{l}\text { Libertad para influir } \\
\text { sobre la definición } \\
\text { de mis objetivos } \\
\text { (19.3 (CM) }\end{array}$ & 0,285 & $-0,325$ & $-0,221$ & 0,125 & $-0,295$ \\
\hline $\begin{array}{l}\text { Poder ser Creativo } \\
\text { (Resolución de pro- } \\
\text { blemas, 11.2; CM) }\end{array}$ & 0,292 & $-0,301$ & $-0,248$ & 0,176 & 0,278 \\
\hline $\begin{array}{l}\text { Recibir información } \\
\text { clara y directa sobre } \\
\text { los Resultados del } \\
\text { Trabajo (Retroali- } \\
\text { mentación del tra- } \\
\text { bajo, P. 11.3; CM y S) }\end{array}$ & 0,25 & $-0,254$ & $-0,197$ & 0,222 & $-0,277$ \\
\hline $\begin{array}{l}\text { Condiciones de tra- } \\
\text { bajo: físicas, como- } \\
\text { didades, seguridad, } \\
\text { P.15.10; (C) }\end{array}$ & 0,273 & $-0,31$ & $-0,224$ & 0,23 & $-0,265$ \\
\hline
\end{tabular}

Fuente: elaboración de los autores. 
Pero el análisis no termina aquí: al evaluar los Estados Psicológicos Críticos, se manifiesta aún más la complejidad que exige pensar en mejorar el bienestar humano.

\subsection{Los Estados Psicológicos Críticos (EPC) y su rol de mediación}

En el presente apartado se describe la correlación en ambos sentidos: hacia atrás, entre las características del trabajo y los EPC; y hacia adelante: cómo interactúan con los Resultados del Trabajo

- ¿Qué Características del Diseño de la Función Laboral (CDT), estimulan los Estados Psicológicos Críticos (EPC) ${ }^{37}$ ?

En primer lugar, la significatividad es el más importante EPC, en concordancia con Johns, Xie y Fang (1992) y Humphrey, Nahrgang y Morgeson (2007): quienes gestionan RRHH deberían trabajar activamente en las características motivacionales de la función laboral que mayor correlación manifiestan. En este sentido, las personas describen que la significatividad se activa cuando ellos experimentan la necesidad de aplicar una variedad de habilidades y talentos $(-0,403)$, cuando sienten que el puesto es importante para la organización $(0,393)$, pueden ser creativos $(0,391)$, autónomos en distintos sentidos, así como cuando realizan tareas diversas $(-0,326)$. Los indicadores de autonomía que más se destacan son la posibilidad de elegir procedimientos $(0,366)$ y de tomar decisiones según experiencia o conocimientos $(0,365)$.

La misma herramienta de diagnóstico para enriquecer las funciones laborales (HDEFL) fue aplicada sobre 350 personas $(2015,2016)$ en América Latina, aplicando luego modelos de ecuaciones estructurales (SEM) para analizar profundamente las relaciones entre determinadas variables. Los resultados parciales evidenciaron que la significatividad del trabajo está fuertemente condicionada por la variedad de tareas, la importancia del cargo y la retroalimentación; la importancia más que la autonomía incentiva el grado de responsabilidad asumido. Finalmente, las variables que favorecen el grado de conocimientos de los resultados alcanzados no son las variables de interacción social, sino el hecho de disponer de información directa y clara, poder autoevaluarse y corregirse y ser conscientes de la importancia del cargo.

37 El paper en cuestión describe asimismo en detalle la construcción del modelo. Genoud, M. A.; Broveglio, G. y Picasso, E. (2018). 
Ahora bien: cuando los trabajadores experimentan que el puesto es importante para la organización, se activan los tres estados psicológicos: la significatividad $(0,393)$, la preocupación por conocer los resultados del trabajo $(0,319)$ y el grado de responsabilidad asumido $(0,297)$. Sucede algo similar, pero con menores correlaciones cuando pueden autoevaluarse y corregirse, cuando reciben información clara y directa sobre los Resultados del Trabajo y así como cuando poseen autonomía para tomar decisiones según la experiencia o conocimientos.

La responsabilidad acerca del trabajo realizado presenta correlaciones bajas. Resulta desafiante intentar estimular que los colaboradores asuman mayores responsabilidades, haciéndoles sentir la importancia de la función, generando tareas diversas y delegando mayores grados de autonomía en la toma de decisiones, cuando acrediten experiencia o conocimientos.

Finalmente, en relación con el tercer estado psicológico crítico, los trabajadores expresan que se mejoraría el grado de conocimiento de sus resultados, si además de ser conscientes de la importancia del cargo (0,319), pudiesen evaluarse y corregirse $(0,306)$, si recibiesen información directa y clara de sus desempeños $(0,281)$ y si dispusieran de mayor autonomía para tomar decisiones según sus experiencias y conocimientos $(0,278)$.

De todos los indicadores que ayudan a entender el peso que tienen las $c a-$ racterísticas sociales en los estados psicológicos, la retroalimentación que proviene de consultas a terceros, la interacción con clientes o con personas externas, no mejoran el grado de responsabilidad asumida ni mejoran el nivel de conocimientos acerca de los resultados alcanzados. No es un tema menor si se tiene en cuenta la importancia que posee el trabajo en equipo en la actualidad laboral. En otras palabras, se ponen de manifiesto aspectos a trabajar para enriquecer el desempeño. La tabla $n^{\circ} 9$ amplía la información. 
Tabla nº 9: Características del Diseño de la Función Laboral (CDT y Estados Psicológicos Críticos (EPC)

\begin{tabular}{|c|c|c|c|}
\hline \multirow{2}{*}{$\begin{array}{l}\text { Características del Diseño de la Función } \\
\text { Laboral (CDT): Características Motivacio- } \\
\text { nales (CM); Sociales (CS) y Contextuales } \\
\text { (CS }\end{array}$} & \multicolumn{3}{|c|}{$\begin{array}{c}\text { Estados Psicológicos Críticos } \\
\text { (Correlación Spearman) }\end{array}$} \\
\hline & $\begin{array}{l}\text { Significativi- } \\
\text { dad del tra- } \\
\text { bajo (15.1) }\end{array}$ & $\begin{array}{l}\text { Grado de res- } \\
\text { ponsabilidad } \\
\text { del trabajo } \\
(15.2)\end{array}$ & $\begin{array}{l}\text { Conocimien- } \\
\text { tos de los re- } \\
\text { sultados } \\
(15 \cdot 3)\end{array}$ \\
\hline $\begin{array}{l}\text { Aplicación de variedad de las habilidades y } \\
\text { talentos (8) }\end{array}$ & $-0,403$ & $-0,161$ & $-0,156$ \\
\hline $\begin{array}{l}\text { Variedad de las tareas: diversidad versus } \\
\text { monotonía ( } 7.3)\end{array}$ & $-0,326$ & 0,263 & $-0,181$ \\
\hline $\begin{array}{l}\text { Poder ser Creativo (Resolución de proble- } \\
\text { mas, 11.2; CM) }\end{array}$ & 0,391 & 0,157 & 0,184 \\
\hline $\begin{array}{l}\text { Recibir información clara y directa sobre } \\
\text { los Resultados del Trabajo (Retroalimenta- } \\
\text { ción del trabajo, 11.3; CM y CS) }\end{array}$ & 0,251 & 0,208 & 0,281 \\
\hline Poder autoevaluarse y corregirse (11.4, CM) & 0,28 & 0,236 & 0,306 \\
\hline $\begin{array}{l}\text { Autonomía para decidir los procedimien- } \\
\text { tos (11.1) }\end{array}$ & 0,366 & 0,145 & 0,192 \\
\hline $\begin{array}{l}\text { Autonomía para tomar decisiones según } \\
\text { experiencia o conocimientos (19.4; CM) }\end{array}$ & 0,365 & 0,254 & 0,278 \\
\hline $\begin{array}{l}\text { Libertad para influir sobre la definición de } \\
\text { mis objetivos (19.3 (CM) }\end{array}$ & 0,355 & 0,199 & 0,257 \\
\hline $\begin{array}{l}\text { Exigencia en la formación o grado de es- } \\
\text { pecialización profesional (9; CM) }\end{array}$ & 0,309 & 0,122 & 0,049 \\
\hline $\begin{array}{l}\text { Grado de importancia del puesto en la } \\
\text { organización }(14,4 ; \mathrm{CM})\end{array}$ & 0,393 & 0,297 & 0,319 \\
\hline Interdependencia y Apoyo (14.5; CS) & 0,143 & 0,054 & 0,035 \\
\hline $\begin{array}{l}\text { El trabajo requiere consultar información } \\
\text { de otras personas (Retroalimentación de } \\
\text { otros, } 14.7 ; \mathrm{CS} \text { ) }\end{array}$ & 0,171 & 0,16 & 0,117 \\
\hline $\begin{array}{l}\text { Interacción fuera de la organización } \\
(14.6 ; \mathrm{CS})\end{array}$ & 0,15 & 0,068 & 0,141 \\
\hline $\begin{array}{l}\text { Condiciones de trabajo: físicas, comodi- } \\
\text { dades, seguridad, P.15.10; (C) }\end{array}$ & 0,198 & 0,165 & 0,172 \\
\hline
\end{tabular}

Fuente: elaboración de los autores. 
- ¿Qué EPC (en términos de significatividad, responsabilidad y grado de conocimiento de los resultados laborales) condicionan los Resultados del Trabajo (RT) (en sus indicadores de comportamiento (RC), actitudes (RA) y bienestar (RB)?

Nuevamente la significatividad se manifiesta como el estado psicológico más importante si se desean estimular resultados de comportamiento, actitudinales, y de bienestar. Más concretamente, los colaboradores mencionan su importancia si se desea activar el grado de satisfacción de quienes quieren crecer $(0,672)$, el nivel de satisfacción del trabajo $(0,651)$ y la motivación interna $(-0,416)$. En relación con el bienestar, las personas que incrementan la significatividad laboral expresan sensaciones de agrado $(-0,52)$, le atribuyen mayor sentido de importancia al trabajo realizado $(-0,512)$ y experimentan menor sensación de aburrimiento $(0,477)$ y estrés $(-0,307)$.

Como se anticipó, el sentido de la responsabilidad asumido no resulta sencillo de estimular y tampoco se correlaciona tan contundentemente con los RT; en términos generales lo que las personas expresan es que, al asumir mayores responsabilidades, aumenta el grado la satisfacción del trabajo $(0,377)$, sienten mayores posibilidades de crecimiento $(0,372)$ y experimentan mayor importancia respecto de su actividad $(0,323)$.

También disponer de conocimientos acerca de los $R T$ mejora la satisfacción del trabajo $(0,437)$, alienta la motivación interna $(0,351)$ y contribuye a combatir el sentido de trivialidad y agrado respecto al trabajo $(0,289)$.

Finalmente, la herramienta brinda información adicional respecto a cómo se relacionan los tres EPC, con los sentimientos recurrentes en términos de bienestar y a la identificación de factores de presión. En general, las personas expresan que ni la responsabilidad del trabajo ni el grado de conocimiento de los resultados alcanzados, generan unívoca y taxativamente sentimientos de ansiedad o inseguridad, ni se vinculan con presión temporal, la sobrecarga de tareas o la presión por interactuar con el cliente. La tabla $\mathrm{n}^{\circ} 10$ amplía la información. 
Tabla nº 10: Estados Psicológicos Críticos (EPC) y Resultados del Trabajo (RT) en términos de Comportamiento (RC), Actitudes (RA) y Bienestar (RB)

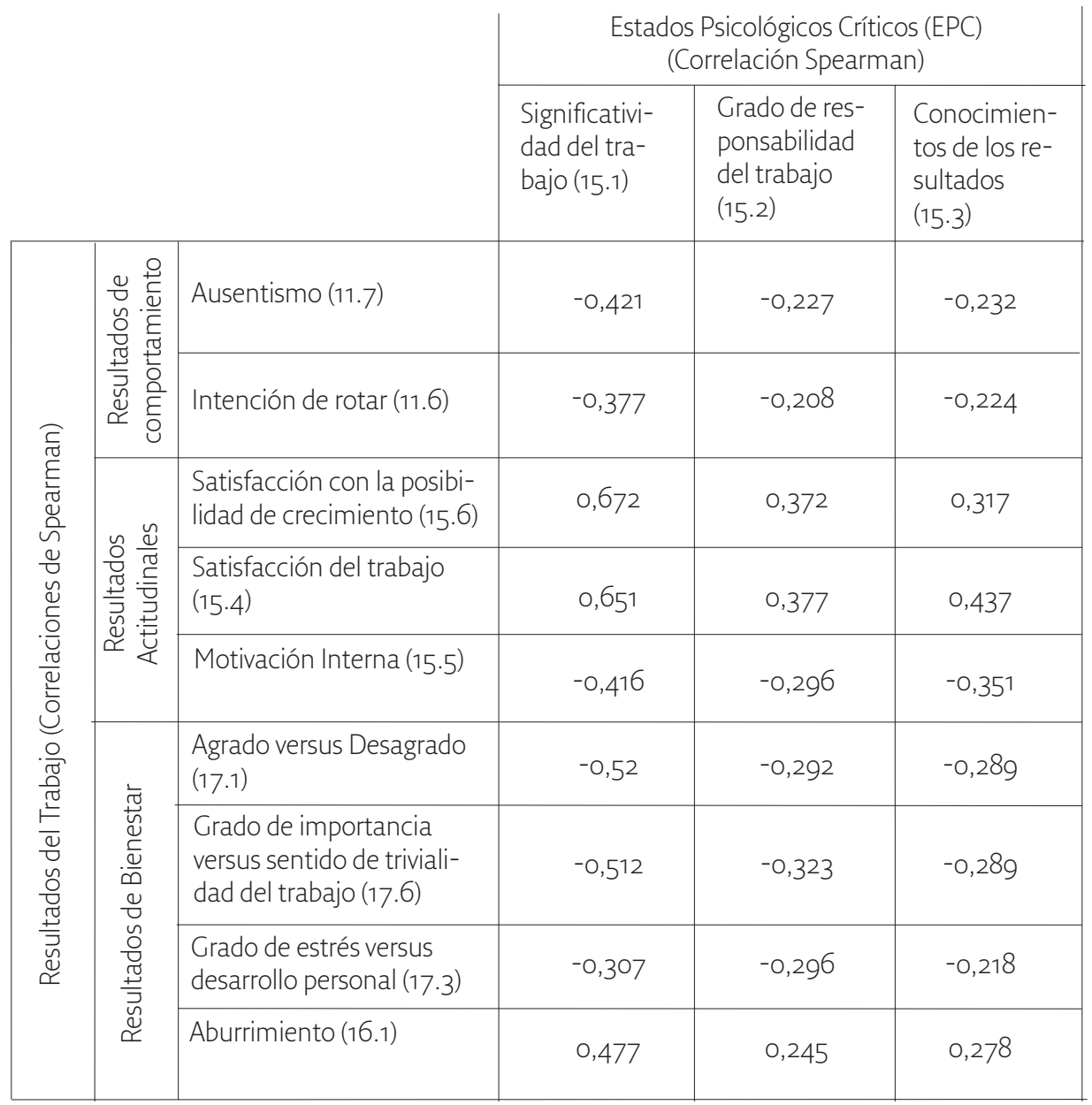

Fuente: elaboración de los autores. Por razones de espacio se han colocado las variables independientes en las filas. 


\section{CONCLUSIONES: PROPUESTAS PARA MEJORAR LOS RESUlTADOS DEL TRABAJO}

Como se ha anticipado, la presente investigación se enmarca en un proyecto global cuyo propósito es reelaborar un instrumento que permita diagnosticar cómo enriquecer la realidad laboral, con miras a mejorar los RT, en términos de comportamiento, de actitudes (lo cual abarca entender diferencialmente grados de satisfacción y motivación de los trabajadores) y de bienestar.

La idea de diagnosticar para poder sugerir prácticas de gestión es sumamente compleja: en primer lugar, porque las variables a incluir deberían considerar aspectos relevantes del propio ambiente laboral en América Latina; asimismo, gran parte de la investigación ha demostrado que los efectos positivos de un resultado a menudo resultan en efectos negativos sobre otros resulta$\operatorname{dos}^{38}$. A pesar de su enfoque interdisciplinario, sigue habiendo lagunas en la cobertura de construcción y medición ${ }^{39}$, lo que sugiere la necesidad de investigar el trabajo de diseño integrador adicional y su conceptualización ${ }^{40}$.

El primer objetivo de este paper es contribuir a responder qué $C D T$ de la función laboral deberían gestionarse con miras a aumentar los Resultados de Comportamiento, los Actitudinales y los de Bienestar. Con miras a facilitar la lectura, se presentan las conclusiones siguiendo el orden del modelo:

Para mejorar los Resultados de Comportamiento, las personas evidencian que se reduce el ausentismo si experimentan que pueden ser creativos $(-0,303)$, si poseen autonomía en términos de libertad para opinar $(-0,300)$; si existen buenas condiciones de trabajo $(-0,277)$ y si reciben información clara y directa de los resultados del trabajo $(-0,256)$. De igual forma para disminuir la intención de rotar en el trabajo, se deberían mejorar las condiciones de trabajo ($0,346)$, fomentar mayor autonomía de expresión $(-0,279)$ y dar feedback ($0,251)$.

En relación con los resultados actitudinales, para lograr apalancar la Satisfacción del trabajo, se debería dar feedback sobre los resultados del trabajo $(0,412)$, ampliar la libertad para opinar $(0,374)$, generar espacios para que los

38 Campion, M. A.; Mumford, T. V.; Morgeson, F. P. \& Nahrgang, J. D. (2005).

39 Edwards, J. R.; Scully, J. A. y Brtek, M. D. (2000).

40 Parker, S. K.; Morgeson, F. P. y Johns, G. (2017). 
trabajadores experimenten que pueden ser creativos $(0,368)$ y otorgar mayor autonomía para tomar decisiones $(0,368)$.

Si se quisiera mejorar la Satisfacción con el superior se debería trabajar en dar feedback sobre los resultados del trabajo $(0,406)$ y promover el sentido de identidad con respecto a la tarea desarrollada $(0,329)$.

Si se desea mejorar la Satisfacción con los compañeros, el superior inmediato debería dar feedback claro y directo sobre los resultados $(0,305)$ y estimular de libertad para opinar $(0,274)$.

La Satisfacción con el Sueldo mejora si los trabajadores reciben información clara y directa sobre los resultados $(0,299)$ y si poseen libertad para opinar $(0,233)$.

Si se quisiera estimular la Satisfacción con la posibilidad de crecimiento, se deben generar espacios creativos de trabajo $(0,456)$, otorgar autonomía para tomar decisiones $(0,407)$, permitir que participen en la definición de los objetivos $(0,388)$ y ofrecer un trabajo con tareas diversas y no repetitivas $(0,384 \mathrm{y}$ $0,381)$.

En lo que hace a la Motivación interna se debería reforzar el sentido de la identidad de la tarea $(0,329)$, dar cuenta de la importancia del puesto $(-0,290)$, fomentar la autonomía para tomar decisiones $(0,297)$ y brindar retroalimentación del trabajo $(0,288)$.

Por su parte, la organización debería tener en cuenta que las condiciones contextuales físicas del trabajo, constituyen un componente clave en los resultados actitudinales: se presenta como un factor higiénico muy fuerte que compensa la insatisfacción respecto del sueldo $(0,468)$, mejora la relación con el superior $(0,401)$, con el trabajo $(0,334)$, con los compañeros $(0,324)$, así como activa la motivación interna $(-0,25)$. Cabe resaltar entonces esta aparente contradicción teórica con los postulados de Modelo de Herzberg et al. (1959): los datos revelan particularidades de estos contextos laborales de América Latina: las inequidades en términos de condiciones estructurales (acceso a trabajos mas significativos, a la salud, a la vivienda, a la educación, a la estabilidad en términos de derechos laborales) son muy difíciles de superar, pero la posibilidad de que se mejoren las condiciones contextuales, aunque sea en el trabajo real, alienta la motivación de los trabajadores.

Finalmente, para mejorar los resultados de bienestar, se debería aumentar la autonomía para decidir con base en los conocimientos y experiencias que acrediten los trabajadores, pues favorece el sentido de importancia del trabajo 
$(-0,362)$, opaca la sensación de aburrimiento $(-0,356)$, estimula el sentimiento de agrado hacia el trabajo $(-0,336)$ y disminuye el estrés $(-0,252)$. Simultáneamente, si se otorga autonomía para decidir los procedimientos, se atenúa la ansiedad (0,247).

No es un dato menor que el trabajo es, de manera contundente, un factor importante en la vida de las personas: el 69\% lo afirma. Sin embargo, los resultados de bienestar son complejos, en posteriores publicaciones se ampliará su análisis. Cabe aclarar que el instrumento de medición ha evidenciado que su complejidad radica en que la gente simultáneamente experimenta sensaciones ambivalentes y concurrentes: la ansiedad por liberarse del trabajo convive con el temor a perderlo, aún más en tiempos de desempleo o alta incertidumbre; tener trabajo contribuye al desarrollo, aún para los más jóvenes, pero las condiciones en las que se trabaja y vive, también da cuenta que el trabajo es una fuente de estrés.

Asimismo, respecto del bienestar, resta precisar lo que el instrumento aporta en relación a identificación o no de factores de presión en el trabajo: no se evidencia de manera contundente, el padecimiento respecto a la presión temporal, la sobrecarga o la interacción con el cliente ${ }^{41}$; cabe preguntarse si los datos que revelan la resistencia a asumir mayores grados de responsabilidad en el trabajo, no contribuyen a explicar que los trabajadores desestimen la injerencia de estos factores de presión. Retomando la primera razón expresada al comienzo del paper, se abre un espacio para reflexionar si los problemas coyunturales que viven las personas en América Latina, no propician una forma de transitar la vida en la cual las presiones se naturalizan, las normas se pasan por alto, sin desestimar que más allá de las fronteras laborales, se viven situaciones de inequidad, de violencia, de incertidumbre, que alteran el bienestar de las personas.

Resta sintetizar el segundo objetivo del paper, lo cual implica precisar si los estados psicológicos críticos impactan de igual forma sobre los resultados del trabajo. Los tres EPC impactan más potentemente en los RT, que las CDT por separado; son sólo tres y concentran un nivel de injerencia más fuerte, liderando la significatividad. En este sentido quienes gestionan RRHH deberían, trabajar activamente en generar trabajos en los cuales se demande variedad de habilidades y talentos, se vivencie la importancia de la función para la organización, se fomente la creatividad y se propicie la autonomía. Es contundente

41 Antes de realizar el procedimiento de análisis aplicando la metodología de correlación canónica se realizaron análisis descriptivos, segmentando entre otras variables por edad y género. 
la correlación entre la significatividad y los resultados actitudinales (grado de satisfacción con el crecimiento, con el trabajo y con la motivación interna); con los de bienestar (mayor sensación de agrado y menor estrés) así como su contribución a los resultados de comportamiento (baja del ausentismo).

Adicionalmente, la herramienta da cuenta que cuando los trabajadores experimentan que el puesto es importante para la organización, se activan los tres estados psicológicos.

Si bien se ha anticipado que es difícil activar el grado de responsabilidad a través de las CDT, quienes gestionan enfrentan el desafío de enriquecer las funciones laborales, ampliando el margen de responsabilidad porque esto facilitaría el grado de satisfacción laboral y contribuiría a sumar importancia a la labor desempeñada. Lo mismo sucedería si se mejorasen los niveles de conocimiento acerca de los resultados laborales.

A modo de cierre, recapitulando respecto a la información que proveen los trabajadores con respecto a las CDT, poseer autonomía (en ámbitos que involucran la toma decisiones, el poder hacer uso del conocimiento o la experiencia; la elección de procedimientos o la libertad de expresión), poder ser creativos, así como recibir información clara y directa de los resultados del trabajo, mejora el bienestar, atenúa también los resultados de comportamiento negativos pero no alcanza para activar siempre todos los resultados actitudinales del trabajo.

Hasta aquí se ha presentado una síntesis del diagnóstico correspondiente a la fase de la investigación 2019.

El diseño de las funciones laborales juega un papel central en las personas, los equipos y el funcionamiento de la organización. A partir de la década del 80, se afianzaron las industrias de servicios, principalmente orientadas al conocimiento, dejando claro que la clave del éxito organizacional está ligado a proveer alta calidad de servicios y a la creación de productos innovadores, a menudo garantizado mediante el uso de diversos tipos de equipos (virtuales, multifuncionales, multijerárquicos, auto gestionados, etc.). La globalización y el continuo avance tecnológico, han afectado a todas las industrias, lo que lleva a algunos autores a sugerir que las estructuras organizacionales se han aplanado $^{42}$. En tal ambiente, la colaboración, la adaptabilidad y la resolución de problemas de manera dinámica, son particularmente importantes.

42 Friedman, T. L. (2005). 
De cara al futuro, en la fase 2020, la herramienta ha incorporado nuevas variables y ya está disponible online $\mathrm{e}^{43}$ a fin de continuar mejorando la forma de abordar temas que poseen prioridad en términos de propuestas de gestión. En este sentido, se han incluido aspectos relacionados con la flexibilidad laboral, las características sociales y las diferencias percibidas en términos del tipo de vínculo laboral que posea el trabajador con la empresa.

Con base en el análisis de experiencias de trabajo en equipo en el sector bancario argentino, en circunstancias en las que se utilizan prácticas de metodologías ágiles, las personas han evidenciado que es difícil compatibilizar el problema de la medición meritocrática individual (que usualmente activa la competencia interna) en detrimento de la cooperación asociada al trabajo en equipo. Ergo, las reformas en el desarrollo de las funciones laborales, demandarían re trabajar las CDT y utilizar otros sistemas de evaluación de desempeño, contemplando el devenir del resultado grupal más que el individual.

En línea con la opinión de varios autores, el hecho que aún no se haya desarrollado una teoría articulada del significado del trabajo ${ }^{44}$, sumado a la necesidad de no minimizar las diferencias situacionales de significado y de identidad local en el trabajo ${ }^{45}$, se cree que es importante profundizar el análisis de algunas variables más que otras. Gaggiotti explica que justamente, frente a la difícil tarea de encontrar una definición única del término trabajo ${ }^{46}$, cada autor adopta una definición en función de los objetivos del estudio que procura realizar $^{47}$. En la fase actual de la investigación con la HDEFL, se busca profundizar las diferencias de significación de cada función laboral, ampliando las categorías dentro de la variable tipo de vínculo laboral... la idea es comprender a los empleados asalariados en relación de dependencia y a otros trabajadores cuyas formas de contratación evidencian condiciones de mayor precarización, pues constituye un área de interés transdisciplinaria para la sociología laboral en América Latina.

43 http://www.surveytech.com.ar/surveytech/index.php/675857?lang=es

44 Harpaz, I. y Snir, R. (2003), MOW (1987).

45 Hofstede, G. (1991); Trompenaars, F. y Hampden-Turner, C. (1998); y Genoud, M. A. (2007) han respaldado empíricamente que habría una relación directa en las prácticas laborales en tanto éstas son definidas con base en los condicionantes del país en el cual se desarrollen. Genoud, M. A. (2007) ahondó la idea de profundizar que, lejos de imponer condiciones homogeneizantes, la globalización ha revitalizado las diferencias culturales.

46 Noon, M. \& Blyton, P. (2002).

47 En el trabajo citado de este autor, consideró trabajo al empleo asalariado (Ruiz- Quintanilla, S. A, \& Claes, R. (2000), tal cual el MOW lo ha definido para sus estudios. MOW (1987). 


\section{BIBLIOGRAFÍA}

Agulló Tomás, Esteban y Ovejero Bernal, Anastasio (Coord.) (2001), Trabajo, individuo y sociedad. Perspectivas psicosociológicas sobre el futuro del trabajo, Ediciones Pirámide (Grupo Amaya, S. A.), Madrid.

Birnbaum, Philip; Farh, Jiing-lih \& Wong, Gilbert (1986), "The Job Characteristics Model in Hong Kong", Fournal of Applied Psychology, vol. 71, $\mathrm{n}^{\mathrm{o}} 4$, pp.598-605.

Breaugh, James A. (1985), "The Measurement of Work Autonomy”, Human Relations, vol. 38, $\mathrm{n}^{\circ}$ 6, pp. 551-570.

Brook, Judith B. (1989), "Exploring the Meaning of Work and Nonwork", Fournal of Organizational Behavior, vol. 10, n² 2, pp. 169-178.

Burke, Ronald J. (2002), Workaholism in Organizations: Antecedents and Consequences, Routledge, London.

Campion, Michael A. (1988), "Interdisciplinary Approaches to Job Design: A Constructive Replication with Extensions", fournal of Applied Psychology, vol. 73, $\mathrm{n}^{\circ} 3$, pp. 467-481.

Campion, Michael A.; Medsker, Gina J.; Higgs, A. Catherine (1993), "Relations between Work Group Characteristics and Effectiveness: Implications for Designing Effective Work Groups", Personnel Psychology, vol. 46, n ${ }^{\circ}$ 4, pp. 823-850.

Campion, Michael A.; Mumford, Troy V.; Morgeson, Frederick. P. \& Nahrgang, Jennifer D. (2005), "Work Redesign: Eight Obstacles and Opportunities", Human Resource Management, vol. 44, $\mathrm{n}^{\circ}$ 4, pp. 367-390. https://doi.org/10.1002/hrm.20080

Campion, Michael A. y Thayer, Paul W. (1985), "Development and Field Evaluation of an Interdisciplinary Measure of Job Design", fournal of Applied Psychology, vol. 70, n 1, pp. 29-43.

Comisión Económica para América Latina y el Caribe (CEPAL) y la Organización Internacional del Trabajo (OIT) (2019), "Coyuntura Laboral en América Latina y el Caribe. El futuro del trabajo en América Latina y el Caribe: antiguas y nuevas formas de empleo y los desafíos para la regulación laboral", no 20. Recuperado 05/2019 en https://www.cepal.org/es/publicacio- 
nes/44604-coyuntura-laboral-america-latina-caribe-futuro-trabajo-americalatina-caribe

Conover, William. J. (1980), Practical Nonparametric Statistics, Wiley \& Sons, Nueva York.

Davis, Louis E. y Taylor, James C. (1979), “Overview”, en Davis, Louis E. and Taylor, James C. (Eds.), Design of fobs, Goodyear Publishing Company, Santa Monica, pp. 10-21.

De la Garza Toledo, Enrique (2000), Tratado Latinoamericano de Sociología del Trabajo, Primera Reimpresión. Facultad Latinoamericana de Ciencias Sociales, Universidad Autónoma Metropolitana y Fondo de Cultura Económica, El Colegio de México, México.

De la Garza Toledo, Enrique (Coord.) (2006), Teorías sociales y estudios del trabajo: Nuevos enfoques, Anthropos, Barcelona.

De la Garza Toledo, Enrique y Neffa, Julio C. (Coord.) (2001), El futuro del trabajo. El trabajo Colección Grupos de Trabajo, Consejo Latinoamericano de Ciencias Sociales (CLACSO), Buenos Aires.

Diaz de Rada, Vidal. (2001) Diseño y elaboración de cuestionarios para la investigación comercial, ESIC, Madrid.

Drenth, Pieter J.D. (1991), “Work Meanings: a Conceptual, Semantic and Developmental Approach", European Work and Organizational Psychologist, vol. $1, \mathrm{n}^{\circ} 2 / 3$, pp. 125-133.

Edwards, Jeffrey R.; Scully, Judith A. y Brtek, Mary D. (1999), The Measurement of Work: Hierarchical Representation of the Multimethod Job Design Questionnaire", fournal of Applied Psychology, vol. 52, n² 2, pp. 305-334.

Edwards, Jeffrey R.; Scully, Judith A. y Brtek, Mary D. (2000), "The Nature and Outcomes of Work: A Replication and Extension of Interdisciplinary Work-Design Research", fournal of Applied Psychology, vol. 85, nº 6, pp. 860868.

Fortea, Miguel A.; Fuertes, Francisco y Agost, María R. (1994), "Evaluación del Modelo Motivacional de las Características del Puesto, a partir de una Muestra Variada", Psicología del Trabajo y de las Organizaciones, vol. 10, $\mathrm{n}^{\circ}$ 29, pp. 32-52.

Fried, Yitzhak, y Ferris, Gerald R. (1987), The Validity of the Job Characteristics Model: A Review and Meta-Analysis", Personnel Psychology, vol. 40, $\mathrm{n}^{\mathrm{o}} 2$, pp. 287-322. 
Friedman, Thomas L. (2005), The World is Flat: A Brief History of the Twenty-First Century, Farrar, Straus, and Giroux, New York.

Gaggiotti, Hugo (2004), “¿Quiénes quieren ser globales? Deslocalización, sentido del trabajo y resistencia a la globalización en los directivos de empresas multinacionales españolas e hispanoamericanas", Revista electrónica de Geografía y Ciencias Sociales. Universidad de Barcelona, vol. VIII, nº 170 (5).

García-Bernal, Javier; Gargallo-Castel, Ana; Marzo-Navarro, Mercedes y Rivera-Torres, Pilar (2005), "Job Satisfaction: Empirical Evidence of Gender Differences", Women in Management Review, vol. 20, n 4, pp. 279-288.

Genoud, María A. (2009), (2007), Imágenes de las Estructuras Laborales en un Mundo Globalizado, Editorial Académica Española, marca comercial de LAP LAMBERT Academic Publishing GmbH \& Co. KG Heinrich-Böcking-Str, Saärbrucken.

Genoud, María A. (2009), “¿Qué objetivos motivacionales buscan satisfacer los argentinos fuera y dentro de sus trabajos? Estudio socio- laboral respecto de los valores argentinos", Revista Sociedad y Economía, vol. 17, pp. 143158. Recuperado en: http://sociedadyeconomia.univalle.edu.co/index.php /sociedad_y_economia/\%20article\%20/view\%20/4181

Genoud, María A.; Broveglio, Gerardo y Picasso, Emilio (2012), "Motivaciones laborales en empresas productivas y servuctivas en CABA y GBA, Argentina", Revista Estudios Gerenciales. Fournal of Management and Economics for Iberamerica, vol. $28, \mathrm{n}^{\circ} 123$, pp. 65-84.

Genoud, María A.; Broveglio, Gerardo y Picasso, Emilio (2014), Gestión estratégica del capital humano diverso, Buenos Aires: Aplicación Tributaria.

Genoud, María A.; Broveglio, Gerardo y Picasso, Emilio (2018), “¿Cómo estimular la significatividad, la responsabilidad y el nivel de conocimientos? Herramienta de diagnóstico para enriquecer las funciones laborales", Ciencias Administrativas, vol. 12, 025. DOI: https://doi.org/10.24215/23143738e025

Gracia, Francisco J.; Martín, Pilar, Rodríguez, Isabel y Peiró, José M. (2001), "Cambios en los componentes del significado del trabajo durante los primeros años de empleo: Un análisis longitudinal”, Anales de Psicología, vol. $17, \mathrm{n}^{\circ} 2$ (diciembre), pp. 201-217.

Grant, Adam M.; Fried, Yitzhak \& Juillerat, Tina (2010), "Work Matters: Job Design in Classic and Contemporary Perspectives", In Zedeck, Sheldon (Ed.), APA Handbook of Industrial and Organizational Psychology, vol 1: Buil- 
ding and Developing the Organization, American Psychological Association, Washington, pp. 417-453.

Griffin, C. William (Ed.) (1982), Teaching Writing in All Disciplines, Jossey-Bass Inc, San Francisco.

Guzzo, Richard A. y Dickson, Michael W. (1996), "Teams in Organizations: Recent Research on Performance and Effectiveness", Annual Review of Psychology, vol. 47, pp. 307-338.

Hackman, J. Richard (1987), “The Design of Work Teams”, In Lorsch, Jay (Ed.), Handbook of Organizational Behavior, Prentice-Hall, , Englewood Cliffs, pp. 315- 342.

Hackman, J. Richard y Lawler, Edward E. (1971), "Employee Reactions to Job Characteristics", Fournal of Applied Psychology Monograph, vol. 55, pp. 259-286.

Hackman, J. Richard y Oldham, Greg R. (1974), The fob Diagnostic Survey: An Instrument for the Diagnostic of Jobs and the Evaluation of Job Redesign Projects, National Technical Information Service, Department of Commerce, Springfield.

Hackman, J. Richard y Oldham, Greg R. (1975), "Development of the Job Diagnostic Survey”, fournal of Applied Psychology, vol. 60, pp. 159-170.

Hackman, J. Richard y Oldham, Greg R. (1976), "Motivation through the Design of Work: Test of a Theory", Organizational Behavior and Human Performance, vol. 16, pp. 250-279.

Hackman, J. Richard y Oldham, Greg R. (1980), Work Redesign, Addison-Wesley, Reading.

Hackman, J. Richard y Oldham, Greg R. (2010), "Not what it was and not what it will be: The Future of Job Design Research", fournal of Organizational Behavior, vol. 31, no 2-3, pp. 463-479.

Harpaz, Itzhak \& Fu, Xuanning (2002), "The Structure of the Meaning of Work: A Relative Stability Amidst Change", Human Relations, vol. 55, $\mathrm{n}^{\circ}$ 6, pp. 639-667.

Harpaz, Itzhak; Honig, Benson \& Coetsier, Pol (2002), “A Cross-Cultural Longitudinal Analysis of the Meaning of Work and the Socialization Process of Career Starters", fournal of World Business, vol. 37, no 4, pp. 230244. 
Harpaz, Itzhak, \& Snir, Raphael (2003), "Workaholism: Its Definition and Nature", Human Relations, vol. 56, n 3, pp. 291-319.

Herzberg, Frederic; Mausner, Bernard \& Snyderman, Bárbara, (1959). The Motivation to Work. Wiley, New York/ London.

Hernández Sampieri, Roberto, Fernández Collado, Carlos. y Baptista Lucio, Pilar (2001), Metodología de la investigación, Mc Graw Hill, México.

Hofstede, Geert (1991), Cultures and Organizations: Software of the Mind, McGraw-Hill, London.

Humphrey, Stephen E.; Nahrgang, Jennifer D. y Morgeson, Frederick P. (2007), Integrating Motivational, Social, and Contextual Work Design Features: A Meta-Analytic Summary and Theoretical Extension of the Work Design Literature", fournal of Applied Psychology, vol. 92, n 5, pp. 1332-1356.

Ilgen, Daniel R. y Hollenbeck, John R. (1991), The Structure of Work: Job Design and Roles", In: Dunnette, Marvin D. y Hough, Leaetta M. (Eds.), Handbook of Industrial and Organizational Psychology, vol. 1, Consulting Psychologist Press, Palo Alto, California.

Jackson, Paul R.; Wall, Toby D.; Martin, Robin, y Davids, Keith (1993), "New Measures of Job Control, Cognitive Demand, and Production Responsibility", Journal of Applied Psychology, vol. 78, n 5, pp. 753-762.

Johns, Gary, Xie, Jia L. y Fang, Yongqing (1992), "Mediating and Moderating Effects in Job Design", Fournal of Management, vol. 18, $\mathrm{n}^{\circ}$ 4, pp. 657676.

Johnson, Richard A. \& Wichern, Dean W. (2001), Applied Multivariate Statistical Analysis, Englewood Cliffs, New Jersey.

Karasek, Robert A. (1998), "The New Work Organization, Conductive Production, and Work Quality Policy", In Marmot, Michael (Ed.), Labor Market Changes and Fob Insecurity: A Challenge for Social Wellfare and Health Promotion, WHO/Europe, Copenhagen, pp. 78-105.

Kozlowski, Steve W. J. e Ilgen, Daniel R. (2006), "Enhancing the Effectiveness of Work Groups and Teams", Psychological Science in the Public Interest, vol. $7, \mathrm{n}^{\mathrm{o}} 3$, pp. 77-124.

Kulik, Carol T. y Oldham, Greg R. (1988), "Job Diagnostic Survey”, En Gael, Sidney (Ed.) The Job Analysis Handbook for Business, Industry and Government, John Wiley y Sons, Nueva York, pp. 462-466. 
Meaning of Working International Research Team (MOW) (1987), The Meaning of Working, Academic Press, London.

Morales, Pedro (2006), Medición de actitudes en psicología y educación. Construcción de escalas y problemas metodológicos, Universidad Pontificia Comillas, Madrid.

Morgeson, Frederick P. y Humphrey, Steven E. (2006), “The Work Design Questionnaire (WDQ): Developing and Validating a Comprehensive Measure for Assessing Job Design and the Nature of Work", Fournal of Applied Psychology, vol. 91, nº 6, pp. 1321-1339.

Morgeson, Frederick P. y Humphrey, Steven E. (2008), “Trabajo y equipo de diseño: Hacia una conceptualización más integradora de diseño de trabajo", En Martocchio, Joseph (Ed.), La investigación en el personal y gestión de recursos bumanos, vol. 27, Emerald Group Publishing Limited, Londres, pp. 39-91).

Noon, Mike \& Blyton, Paul (2002), The Realities of Work, Basingstoke, Houndmills/Palgrave, New York.

Parker, Sharon K.; Morgeson, Frederick P. y Johns, Gary (2017), "One Hundred Years of Work Design Research: Looking Back and looking Forward”, Fournal of Applied Psychology, vol. 102, , n³, pp. 403- 420.

Parker, Sharon K. y Wall, Toby D. (2001), "Work Design: Learning from the Past and Mapping a New Terrain”, In Anderson, Neil; Ones, Deniz S.; Sinangil, Handan Kepir y Viswesvaran, Chokalingam (Eds.), Handbook of Industrial, Work and Organizational Psychology: Vol. 1: Personnel Psychology, Sage, London, pp. 90-109.

Parry, Jane (2003), “The Changing Meaning of Work: Restructuring in the Former Coalmining Communities of the South Wales Valleys", Work, Employment \& Society, vol. 17, no 2, pp. 227-246.

Peña, Daniel (2013), Análisis de datos multivariantes, McGraw-Hill España, Madrid.

Pérez, Augusto y Guzmán, Maricela (2015), "Los estudios organizacionales como programa de investigación”, Cinta Moebio, vol. 53, pp. 104123. www.moebio.uchile.cl/53/perez.html

Perretti, Fabrizio y Negro, Giacomo (2006), "Filling Empty Seats: How Status and Organizational Hierarchies Affect Exploration Versus Exploitation in Team Design", Academy of Management fournal, vol. 49, n 4, pp. 759-777. 
Ros, María; Schwartz, Shalom H. \& Surkiss, Shoshana (1999), "Basic Individual Values, Work Values, and the Meaning of Work", Applied Psychology: An International Review, vol. 48, $\mathrm{n}^{\circ}$ 1, pp. 49-71. https://doi.org/10.1111 /j.1464-0597.1999.tb00048.x

Ruiz-Quintanilla, S. Antonio, \& Claes, Rie (2000), "Mow Research Programs", In Katz, Jerome A. (Ed.), Databases for the Study of Entrepreneurship, JAI, Amsterdam-New York, pp. 335-391.

Schwartz, Shalom H. (1992), "Universals in the Content and Structure of Values: Theory and Empirical Tests in 20 Countries", In Zanna, Mark (Ed.), Advances In Experimental Social Psychology, vol. 25, Academic Press, New York, pp. 1- 65 .

Schwartz, Shalom H. (2005a), "Basic Human Values; Their Content and Structure across Countries", In Tamayo, Alvaro y Porto, Juliana B. (Eds.) (2005), Valores e Trabalho, Brasilia: Editora Universidad de Brasilia, Chapter 1 .

Schwartz, Shalom H. (2005b), "Robustness and Fruitfulness of a Theory of Universals in Individual Values", In Tamayo, Alvaro y Porto, Juliana B. (Eds.) (2005), Valores e Trabalho, Editora Universidad de Brasilia, Brasilia, Chapter 2.

Sims, Henry P.; Szilagyi, Andrew D. y Keller, Robert T. (1976), "The Measurement of Job Characteristics", Academy of Management fournal, vol. 19, $\mathrm{n}^{\circ} 2$, pp. 195-212.

Stewart, Greg L. (2006), "A Meta-Analytic Review of Relationships between Team Design Features and Team Performance", fournal of Management, vol. 32, no 1, pp. 29-54.

Sullivan, Pat M. (2003), Work with Meaning, Work with foy: Bringing your Spirit to any fob, Sheed \& Ward, Lanham.

Tang, Thomas L.; Furnham, Adrian \& Davis, Grace M. (2002), "The Meaning of Money: The Money Ethic Endorsement and Work-Related Attitudes in Taiwan, the USA and the UK", Journal of Managerial Psychology, vol. $17, \mathrm{n}^{\circ} 7 / 8$, pp. 542-563.

Trompenaars, Fons y Hampden-Turner, Charles (1998), Riding the Waves of Culture: Understanding Cultural Diversity in Global Business, McGraw Hill, New York. 
Wingu, (2016), Manual de Metodologías Ágiles, elaborado en colaboración con el GBA, Recuperado 16/06/2020 https://www.coursehero.com/file/49130152 /manual-de-metologias-agiles-finalpdf/ 
